\title{
Italique
}

Poésie italienne de la Renaissance

XX $\mid 2017$

Varia

«Adonis et berger et chasseur tout ensemble»: un mito ibrido e la sua fortuna nella bucolica rinascimentale

\section{Carlo Caruso}

\section{OpenEdition}

Journals

Édition électronique

URL : http://journals.openedition.org/italique/465

DOI : $10.4000 /$ italique.465

ISSN : 1663-4438

Éditeur

Librairie Droz

\section{Édition imprimée}

Date de publication : 1 octobre 2017

Pagination : 261-276

ISBN : 978-2-600-05818-6

ISSN : 1423-3983

\section{Référence électronique}

Carlo Caruso, « «Adonis et berger et chasseur tout ensemble»: un mito ibrido e la sua fortuna nella bucolica rinascimentale », Italique [En ligne], XX | 2017, mis en ligne le 01 octobre 2019, consulté le 23 janvier 2020. URL : http://journals.openedition.org/italique/465 ; DOI : 10.4000/italique.465 


$$
\text { C A R L O C A R U S O }
$$

\section{«A D O I S ET B ER GER}

ET CHASSEUR TOUT ENSEMBLE»:

$$
\text { U N M T O IB R I O }
$$

E LA SUA FORTUNA

NELLA B U C O L I C A R I N A C I M E N T A L E 



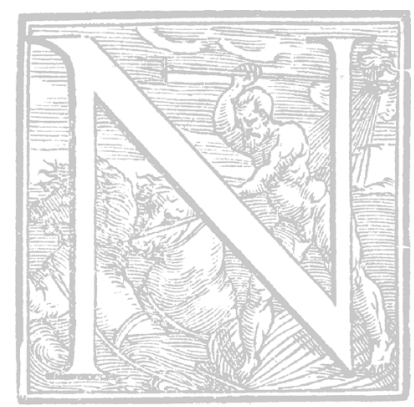

el contesto di un volume dedicato all'esame del codice bucolico tra l'età del Boccaccio e quella del Marino, ho pensato che potesse essere utile offrire un breve resoconto della presenza del mito di Adone nella poesia pastorale di Cinque e di primo Seicento. Il fine è quello di mostrare un fenomeno caratteristico della poesia pastorale (anche se non solo di essa): il suo foggiarsi e combinarsi in vari modi e maniere nell'ambito di generi diversi, non solo dell'egloga ma anche dell'epillio, dell'elegia, e finalmente anche del poema. Non so esimermi da utilizzare ancora una volta - e me ne scuso - l'arguta opinione di un poeta inglese portato come altri mai alla formulazione epigrammatica, il quale, non ancora sedicenne, all'inizio del Settecento commentava:

Non vi è, io credo, numero maggiore di componimenti di ogni sorta che vengano descritti come "pastorali", né numero minore di quelli che sono genuinamente tali. ${ }^{\mathrm{T}}$

Può uno sguardo a certi aspetti della fortuna in età rinascimentale del mito di Adone, che ho definito "mito ibrido" per le ragioni che verrò illustrando, aiutare a intendere, almeno in parte, il fenomeno di tale commistione?

I. Nella versione che godette di maggiore divulgazione, che è poi quella delle Metamorfosi di Ovidio, il mito del bellissimo giovane amato da Venere e imprudentemente desideroso, lui sostanzialmente imbelle, di misurarsi nella caccia al cinghiale - caccia destinata a concludersi con esito tragico, solo in parte redento dalla sua metamorfosi in anemoneè un mito di ordine minore. Quasi lo si potrebbe dire un mero sviluppo narrativo della storia, psicologicamente assai più intensa, della madre Mirra e dell'incestuoso suo rapporto con il padre, di cui Adone è appunto il frutto. L'attenzione rivolta principalmente a Mirra accomuna $i$ lettori del Medioevo, come bene mostrano i commenti allegorici alle Metamorfosi e le riscritture ovidiane "moralizzate". Con il primo Umanesimo, invece, viene crescendo un interesse di natura antiquaria, dovuto in larga parte al recupero dei mitografi tardo-antichi: come si vede, per esempio, nel Boccaccio delle Genealogie deorum 
Gentilium. ${ }^{3}$ E proprio a partire dalle Genealogie boccacciane che la narrazione dell'episodio di Adone (Gen. II 53) si svincola da quello di Mirra (Gen. II 52), in virtù innanzi tutto della trattazione analitica dei miti che caratterizza l'opera boccacciana.

Non più all'ombra del dramma mirrino, il mito adonio sottostà a uno sviluppo indipendente e duplice: complice anche la ricomparsa, un secolo dopo Boccaccio, del Corpus Theocriteum, ovvero dei cosiddetti "Bucolici greci», con gli idilli adonii di Teocrito $(\mathrm{XV})$ e di Bione, e con l'anonimo epigramma Adone morto. Al primo esempio di sviluppo accenno solo rapidissimamente, perché il trattarne condurrebbe il discorso in ambiti che in questa sede non rilevano. Si tratta però del primo, vero e - sia pur per breve tempo - fortunatissimo esempio di rinascita del mito adonio in età umanistica, e come tale impone di essere almeno ricordato. Mi riferisco alla brillante inventio, o piuttosto reinventio, di Giovanni Pontano, il quale riformulò l'aition ovidiano della trasformazione di Adone in anemone per offrirne un'altra, alternativa, di Adone in albero d'arancio: contaminandone cioè il mito con quello del giardino delle Esperidi e inserendone la narrazione in un poemetto sull'agrumicoltura, il De hortis Hesperidum libri duo (che tratta della coltivazione di aranci, limoni e cedri), concepito come moderna risposta, e in certo qual modo anche come supplemento, alle Georgiche virgiliane. ${ }^{4}$ La fortuna di questo audace esperimento, non longeva ma indubbia, si può misurare a riscontro delle molte reazioni entusiastiche al suo postumo apparire a stampa nel isos, delle numerose imitazioni lungo i primi trenta-quarant'anni del Cinquecento, e di un altrettanto autorevole esperimento nel genere eziologico che nel poemetto di Pontano riconobbe apertamente il proprio modello: il Syphilis di Girolamo Fracastoro.'

L'altro esempio di sviluppo del mito, maggiormente pertinente al nostro discorso, presenta Adone come personaggio della poesia pastorale, ovvero come protagonista di storie narrate da pastori. Prescindo qui dalle semplici menzioni del nome di Adone come personaggio esemplare di bellezza insuperata e quasi muliebre nella sua delicatezza imberbe, menzioni che ricorrono con una certa frequenza nella poesia bucolica e anche in altri generi: basti ricordare ancora Boccaccio, nella fattispecie il suo Buccolicum carmen. ${ }^{6}$ Ma faccio eccezione per un unico passo, che dovrebbe essere il primo nelle letterature moderne in cui Adone viene esplicitamente designato come pastore. Tale designazione ricorre nel De 
calamitatibus temporum libri tres (I470) di Battista Spagnoli detto il Mantovano: " pastor formosus Adonis» (I I055). ${ }^{7}$

Al di là del «pastor formosus» del Mantovano, tuttavia, la prima vera caratterizzazione di Adone come personaggio di ambiente pastorale ricorre, salvo errore, nel ventesimo componimento del Lusus di Navagero, l'egloga Damon, che per $i$ riferimenti storici (discernibili attraverso il velo del codice pastorale) risale a immediatamente dopo la sconfitta veneziana di Agnadello, dunque agli anni I509-10. ${ }^{8}$ Invece l'egloga Adone $(X)$ dell'Alamanni, uscita a stampa nelle Opere toscane (Parigi, I532-33), rappresenta la prima sostanziosa apparizione di Adone - si vedrà in quale veste - nella poesia bucolica in volgare. Tornerò su entrambi i testi poco più innanzi. Qui mi limito a osservare che l'Adone pastore viene cosi a figurare accanto all' assai più noto Adone cacciatore, il che giustifica il verso tratto dall'Adonis di Pierre de Ronsard (I563) inserito nel titolo del mio contributo: "Adone ad un tempo pastore e cacciatore».?

2. A tuttiè noto Adone in veste di cacciatore. Diun Adone pastore, invece, si banno scarse, anzi scarsissime notizie, ancorché autorevoli per dignità poetica. Abbiamo, in buona sostanza, un verso dell'egloga x di Virgilio, l'egloga di Gallo, nella quale l'umile contesto pastorale - di cui Adone è dichiarato essere parte - chiede di non essere disprezzato (Buc. X I8):

et formosus ovis ad flumina pavit Adonis.

Il verso virgiliano è quasi certamente ripreso dalla prima egloga di Teocrito, là dove Tirsi rammenta Adone come una delle conquiste, o vittime, di Venere (I I09-I0):

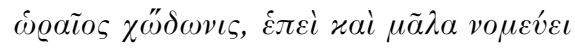

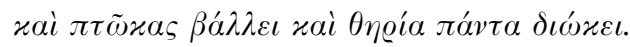

E Adone maturo per l'amore, poi che attende alle greggi E bersaglia le lepri e si dà a inseguire ogni animale.

S'intende poi che $i$ componimenti del Corpus Theocriteum dedicati ad Adone dovettero avvicinare ancor più e quasi cooptare la figura di Adone al genere bucolico. La comparsa di un Adone pastore in Virgilio suscitava però la perplessità del commentatore antico che va sotto il nome di Probo, poiché cosi egli si esprime nella glossa a Buc. X I8: 
Hunc venandi studiosum fuisse, et ab apro interiisse, atque ita plurimis cognitum. Pastorem non invenimus fuisse, sed amatoriam fictam Veneris induxit historiam (in Buc. x I 8).

Che fosse desideroso di cacciare e che venisse ucciso dal cinghiale, è noto a molti. Non si trova che fosse anche pastore; ma questo ispirò l'invenzione della storia degli amori con Venere.

La preoccupazione dello pseudo-Probo circa l'effettivo statuto socialeletterario di Adone è stata fatta propria dagli antropologi dell'età nostra e ha incoraggiato a riconoscere nell'efebo per antonomasia un simbolo di contrasto fra il mondo della caccia e quello della coltivazione e anzi, piu specificamente, della cerealicoltura: dal momento che mitografi e scoliasti greci tardi invitavano a interpretare la vicenda di Adone - vicenda di cicliche morti e reviviscenze stagionali - come allegoricamente allusiva al ciclo del grano. Rammento questo aspetto perché è forse il più noto e vulgato, oltre che oggetto di vivaci dibattiti negli anni Settanta in seguito alla pubblicazione del fortunato e provocatorio volume di Marcel Detienne sugli orti di Adone (Les jardins d'Adonis, 1972) e alle reazioni da esso suscitate. ${ }^{\circ}$ Varie le ipotesi allora proposte, che scartavano quella cerealicola (sostenuta fra gli altri da Frazer nel Ramo d'oro all'inizio del Novecento) e favorivano o l'associazione di Adone alle piante aromatiche e afrodisiache (Detienne), o l'interpretazione della sua storia come esemplare di uno stato incerto e liminare, fra la vita errabonda delle prime società di popoli cacciatori e quella invece più sedentaria delle società dedite alla coltivazione (Piccaluga). Ne risultava un Adone "disadattato", non incasellabile in alcuna delle due occupazioni, e pertanto destinato a soccombere, proprio perché cacciatore sfortunato e coltivatore fallito ad un tempo: non diversamente da figure più o meno assimilabili alla sua, s'intende se osservate in tale ottica antropologica, come Atteone, Ippolito, Perdicca, Ippomene. ${ }^{\text {I }}$ Il verificare quanto vi sia di plausibile in queste teorie è compito che mi è grato lasciare ad altri (di un Adone coltivatore, fra parentesi, io personalmente non ho mai trovato traccia, ed è chiaro che $i$ due elementi del contrasto sopra accennato non stanno sul medesimo piano: il cacciatore è sempre e dichiaratamente tale, mentre il coltivatore è solo immaginabile per via di allusioni indirette e di complessi traslati del codice mitografico). Quando se ne accenna ai colleghi classicisti che di questi aspetti si occupano, il loro volto si atteggia a un sorriso benevolo e anche 
un poco nostalgico, mèmore probabilmente della passata giovinezza, e forse anche di un entusiasmo per quel genere di discussione animata che sembra esser venuto in larga parte meno.

3. Ma gli anni Settanta sono per noi anche gli anni del gran commento di Giovanni Pozzi e Collaboratori all'Adone di Giovan Battista Marino, nel quale, più delle ragioni antropologiche - tenute bensi nella dovuta considerazione, ma anche prudentemente a bada - premeva intendere e risolvere le questioni storico-letterarie. ${ }^{\mathrm{I}}$ E grazie a Pozzi e alla sua scuola si è potuto far chiarezza su come e perché la dicotomia cacciatore-pastore importasse per $i$ poeti del Rinascimento e il loro pubblico: la ragione essendo quella dello scarto, lessicale e stilistico, fra l'epillio mitologico-boschereccio e l'idillio pastorale. Solo cosi credo possa intendersi appieno il verso di Ronsard «Adone ad un tempo pastore e cacciatore». Penso in particolare a un importante contributo sull'Adone di Marino di Alessandro Martini, dove sono presi in esame $i$ precedenti cinquecenteschi del poema mariniano. ${ }^{\text {I }}$ Martini notava come nell'Adonis (IS63) di Ronsard, come del resto già vent'anni prima nelle Stanze nella favola d'Adone (I545) di Ludovico Dolce, ricorrano certe "pastorellerie" estranee ad altri poemetti di metà Cinquecento che narrano l'episodio adonio - quelli di Giovanni Tarcagnota (I550) e di Girolamo Parabosco (I553) - ed estranee anche all'Adone del Marino. ${ }^{\text {I4 }}$ Nelle Stanze nella favola d'Adone di Dolce, osservava Martini, è notevole la scena in cui Venere, per amore del suo Adone, si adatta alla vita rustica dei pastori ed è mostrata intenta a mungere le mammelle «immonde» di capre e agnelle (Dolce, Stanze, $17-18$, 5-8):

Quante fiate in queste parti e in quelle

Del chiuso ovil, quand'esce il sol de l'onde

Menava con Adon le pecorelle

Pe i campi a pascer fiori, herbette e fronde,

E premea or delle capre, or de l'agnelle

Con le celesti man le poppe immonde:

Hor dentro le spelunche incolte e strane

Tosava pur con lui [st. lei] le bianche lane.

$[\cdots]$

Spesso seco prendea lungo viaggio:

E per aspre contrade e pellegrine, 
Per monti e valli più sassose e fere

Con lui seguia le fuggitive fiere.

Pressoché identico, nella sostanza, il passo corrispondente di Ronsard (Adonis, 73-80):

O bien heureux enfant! Dont la belle Cythere, La mere des Amours à toy seul veut complaire! Seulette avecques toy veut tondre les brebis, Et de sa blanche main leur pressurer le pis, Veut mener aveq toy les beufs en pasturage, Esclisser des paniers, \& faire du froumage.

Ma entrambi $i$ passi sono debitori verso il Damon di Navagero (Damon, in Lusus, $x \times 75-77):^{\text {Is }}$

Fortunate puer! Tecum formosa Dione

Una tondet oves, una ad mulctralia ducit,

Atque immunda premit caelestibus ubera palmis.

Fortunato giovane! Accanto a te la bella Venere ora tosa le pecore, ora le conduce alla mungitura, e con le celesti palme ne preme le mammelle immonde.

Navagero sta dunque all'origine della parentesi pastorale, e anzi quasi rusticale, come giustamente notava Martini a proposito di Dolce e Ronsard: il che comporta una percettibile escursione di registro e di tono. Quale il motivo di quest'ansa narrativa pastoral-rusticale? Navagero avrà forse voluto ornare il proprio Damon con una variatio di quanto si legge già nelle Metamorfosi di Ovidio (Met. X 532-37):

Caelo praefertur Adonis.

Hunc tenet, huic comes est adsuetaque semper in umbra indulgere sibi formamque augere colendo per iuga, per silvas dumosaque saxa vagatur fine genus vestem ritu succincta Dianae hortaturque canes...

Alla sede celeste [Venere] preferisce Adone.

Lo ha con sé, facendoglisi compagna, e benché solita indugiare sempre in luoghi ombreggiati, dove cura di farsi più bella, vaga [ora] per gioghi e foreste e luoghi sassosi e spinosi, 
la veste al ginocchio al modo di Diana, e incita i cani.

Come in Ovidio Venere, per amore di Adone, si fa simile alla scattante Diana e rinuncia quindi ai molli agi che le sono cari, cosi in Navagero la dea ci viene mostrata intenta a un sacrificio ulteriore: si adatta non solo alla caccia, ma anche alla rozza e umilissima vita dei pastori. Di qui il compiaciuto utilizzo di termini "bassi" («immunda [...] ubera») e tecnici («mulctralia»).

4. Ho già accennato all'Alamanni e al suo intitolare ad Adone la decima delle sue egloghe: egloghe notevoli per l'adesione al principio trissiniano del verso sciolto come mimesi del "parlato pastorale"; ho anche già ricordato che le egloghe apparvero nelle Opere toscane a Parigi fra il Is32 e il I533. ${ }^{\mathrm{I}}$ Del medesimo periodo, anzi poco prima, tra la fine del Iszo e l'inizio del Is3I, è l'egloga Venere di Girolamo Muzio, pubblicata poi nel I5so, in verso sciolto come quelle dell'Alamanni. ${ }^{\text {I7 }}$ Entrambi $i$ testi, l'Adone di Alamanni e la Venere di Murio, sono dedicati al re di Francia Francesco I, alla cui corte $i$ due poeti erano presenti nei primissimi anni Trenta. Diversi però $i$ modelli: mentre Alamanni organizza la propria egloga come il sesto idillio di Teocrito, inserendovi riferimenti al proprio esilio da Firenze e innestandovi al centro una riscrittura del Lamento su Adone di Bione, Muzio riscrive fantasticamente Ovidio immaginando il re di Francia come Marte attaccato da un cinghiale, alla cui vista Venere, mèmore della morte di Adone, è tramutata per il terrore in statua marmorea (contaminazione evidente - e un poco anche involontariamente comicacon l'episodio, sempre da Ov. Met. $x$, di Pigmalione, che secondo la genealogia mitica è avo di Adone). ${ }^{\text {I } 8}$

Mi permetto qui di tralasciare le questioni relative ai rapporti letterari fra $i$ testi appena ricordati per attirare invece l'attenzione sulle dediche e sul contesto che esse illuminano. E di fatto a partire da questi testi che s'inizia in Francia la fortuna dell'egloga in volgare e anche, entro tale ampio alveo, la fortuna del mito dell'Adone cacciatore e insieme pastore, come appunto specificherà Ronsard imitando Navagero (il quale Navagero, varrà la pena di ricordarlo, era morto proprio alla corte di Francia, a Blois, dove era oratore della Repubblica di Venezia, nel Is29: dunque appena prima del ritorno alla corte francese di Alamanni, reduce dall'assedio di Firenze, e dell'arrivo del Muzio al seguito del 
modenese conte e celebre mecenate Claudio Rangone). ${ }^{19}$ Occorre anche sottolineare che il genere bucolico diviene di li innanzi in Francia il codice letterario regale per eccellenza, con preferenza spiccata per l'éclogue forêtière $o$ éclogue de chasse: dove domina tematicamente la caccia - attività degna di sovrani - ma entro forme e formule bucoliche. $\dot{E}$ anche questo un sintomo del passaggio dalla Francia medievale a quella classica o classicistica, e nella fattispecie il passaggio dalla Francia dei tornei (in uno dei quali perdette la vita il re Enrico II dopo atroci sofferenze) alla Francia delle cacce. Fu detto anzi che Caterina de' Medici, moglie di Enrico II e testimone impotente della morte del marito, instillasse nel figlio Carlo il disgusto per $i$ tornei e ne favorisse invece la passione per la caccia, al punto che il giovane - s'intende anche per vanità - fini per identificarsi con il mitico Adone, come attestano molteplici fonti coeve. ${ }^{20}$ Andò forse un po' troppo in là con l'emulazione, dal momento che mori appena diciottenne (sebbene non cacciando); e come un giovane e sfortunato Adone fu cantato da molti, fra cui Ronsard, che delicatamente lo descrisse come una «Rose Adonienne» prematuramente troncata prima di poter sbocciare (Elegie sur le livre de la chasse du feu Roy Charles IX, pubblicata postuma nel I625); dall'allievo di Ronsard, Claude Binet, in Adonis, ou le Trespas du Roy Charles IX. Eglogue de Chasse (I575); da Guillaume Le Breton con una tragedia intitolata Adonis. Tragedie françoyse (I579). ${ }^{21}$ Sono sufficienti questi esempi, specialmente l'ultimo, per mostrare come in Francia il genere pastorale potesse attingere le vette della gerarchia dei generi sviluppando quell'idea di pastorale héroïque che di per sé suona, del resto volutamente, come un ossimoro, e quasi come una coincidenza degli opposti. Né mancò l'appoggio della formulazione di una nuova teorica dei generi, di cui fu promotore Giulio Cesare Scaligero nella sua Poetica (I56I), autorevolissima in Francia. Ivi il canto amebeo è proclamato «vetustissimum [...] poematis genus», e come tale padre della tragedia e della commedia. ${ }^{22}$

5. La tragedia Adonis di Guillaume Le Breton, di cui ha procurato l'edizione critica un antico allievo di Cesare Segre, Mario Bensi, venne ripubblicata più volte nel tardo Cinquecento e per l'ultima volta nel I6II, appena dopo l'assassinio del re Enrico IV ad opera di François Ravaillac. ${ }^{3}$ Nelle dozzine di opuscoli usciti a seguito di quel ferale e sconvolgente evento - versi, orazioni e sermoni in latino e in francese- 
domina la nota pastorale: a conferma che ancora nel secondo decennio del Seicento il "fantastico regale" continuava a essere predominantemente espresso attraverso quel codice. Il defunto re Enrico IV $\grave{e}$ in genere paragonato al dio Pan: onde risuona più volte il celebre lamento "Il gran dio Pan è morto» a imitazione di Plutarco, De defectu oraculorum, I7 (4I9A-E); ma, benché meno frequentemente, anche il mito di Adone fra la sua comparsa in quella pubblicistica, nella medesima chiave e col medesimo intento: il pugnale di Ravaillac, per esempio, è paragonato alla ferale zanna del cinghiale. ${ }^{24}$ Fatto ancor più significativo, il mito adonio di morte e reviviscenza è ripreso per sottolineare la continuità dinastica tra Enrico IV e il figlio Luigi XIII, quasi i due fossero una medesima persona: riconfermando cosi, e anzi istituzionalizzando proprio in quella circostanza, la formula francese già cinquecentesca di sottolineare la persona decidua del re a contrasto della regalità che invece non muore mai: "Le roi est mort. Vive le roi.». ${ }^{25}$

Entro il contesto del codice pastorale, dunque, il mito adonio continuava in Francia a essere collegato con la persona del re ${ }^{26}$ Si spiega allora come pochi anni più tardi Giovan Battista Marino, approdando a Parigi alla corte della regina madre e vedova di Enrico IV, Maria de' Medici, trovasse nella capitale francese, sia pure con qualche difficoltà, il contesto adatto per fare finalmente uscire a stampa, con dedica ai sovrani francesi, il suo Adone: cresciuto enormemente in quasi trent'anni di lavoro sia pure discontinuo. Occorre sottolineare che l'Adone, già ricordato come oggetto di ammirazione in una lettera di contemporaneo del $1596,{ }^{27}$ era in origine un componimento pastorale che l'autore veniva componendo sotto lo psudonimo pastorale di Carino. ${ }^{28}$ Quanto di questo Adone originario sopravviva nel poema come noi lo conosciamo non è dato sapere. Tra gli anni Novanta del Cinquecento, quando Marino ancora risiedeva a Napoli, e il I623, anno della princeps parigina del poema, quell'iniziale ispirazione bucolica era venuta gonfiandosi e complicandosi per produrre finalmente un opus magnum di oltre quarantamila versi. Crescita sproporzionata, quasi mostruosa se paragonata alle umili premesse, che immaginiamo essere state quelle tipiche di una comune egloga; ma che trovò incentivo in almeno due ambiziosi esperimenti, nei quali l'autorizzazione a produrre componimenti pastorali di lunga, anzi lunghissima lena pareva incoraggiare alla competizione con il modello del poema epico. Il primo esperimento è ben noto agli studiosi di Marino: è Lo stato rustico di 
Giovan Vincenzo Imperiali (I607), gigantesco poema pastorale che a me fece conoscere, in un corso memorabile al Politecnico di Zurigo di tanti anni fa, Ottavio Besomi. ${ }^{29}$ L'altro, che ancora può dirci molto - io credo - sullo sviluppo dell'Adone, è l'Astrée di Honoré d'Urfé, l'altrettanto gigantesco prosimetro pastorale in sessanta libri raccolti in cinque parti, uscito fra il I607 e il I627: il primo grande classico della letteratura francese del Seicento. Marino ne poté solamente vedere a stampa le prime tre parti; ma il suo interesse per d'Urfé è attestato dall'epistolario, e del resto gli editori parigini che tra il I607 e il I627 tennero sotto $i$ torchi le diverse parti dell'Astrée in una confusione quasi inestricabile di edizioni, emissioni e "stati" tipografici parziali sono quei medesimi con i quali Marino ebbe a che fare tra il I6I6 e il I623 per l'analogamente lunga e tormentata lavorazione della princeps dell'Adone, princeps di cui (come per l'Astrée) si troverebbero a stento due esemplari identici. ${ }^{30}$

Queste mutazioni del genere pastorale producono insomma un arazzo "misto" e variopinto, ma non senza una sua riconoscibile linea di sviluppo, una volta che se ne considerino le diverse fasi alla luce dei progressivi mutamenti della storia letteraria e della storia tout court, non più circoscrivibili all'Italia per il periodo dal pieno Cinquecento al primo Seicento. Detto questo, l'Adone come punto di approdo della tradizione qui appena schizzata rimane pur sempre un testo problematico. Emilio Russo, che del poema ha offerto una splendida nuova edizione (e già fare cosa nuova, dopo il commento di Pozzi e Collaboratori, è stato diper sépregio grandissimo), ne ha anche offerto un'interpretazione globale che invita a riconoscere nel testo evidenti scompensi non facilmente riducibili a razionalizzazioni di tipo strutturale o strutturalista. Sia pure nel massimo rispetto e direi nell'indiscussa ammirazione, del resto implicita in ogni suo intervento, per l'opera di Pozzi, Russo ba un poco attenuato l'ambizione a dare piena giustificazione della struttura del poema cosi come ci sta dinanzi. ${ }^{3 \mathrm{I}}$ Per parte mia non posso che consentire; aggiungerei inoltre che, come si intende da altri aspetti della formazione dell'opera, non ultimi quello meccanico della composizione tipografica e quello della limitata accessibilità dei regali dedicatari per via dei forti conflitti in seno alla corte francese di quegli anni, Marino dovette essere costretto a non pochi mutamenti, anche dell'ultima ora, per adeguarsi alle circostanze mutevoli e alle opportunità che l'incertezza via via consentiva. ${ }^{32}$ Ma la vicenda di Adone 
cacciatore e pastore, accolta già nel Cinquecento in Francia all'interno del codice pastorale cortigiano e invocata nel primo Seicento come simbolo di continuità dinastica, gli dovette finalmente offrire il collante ideologico, per cosi dire, con cui tenere insieme la gran massa eterogenea di quel poema nato trent'anni prima come umile egloga.

Carlo Caruso 


\section{Carlo Caruso}

I. Alexander Pope, A Discourse on Pastoral Poetry, in The Poems of Alexander Pope, a cura di J. Butt, London-New Haven, Yale University Press, I96 I-69, I I voll., I, p. 23.

2. Cfr. B. Guthmüller, Ovidio Metamorphoseos vulgare, Firenze, Cadmo, 2008; Id., Mito, poesia, arte. Saggi sulla traduzione ovidiana nel Rinascimento, Roma, Bulzoni, I 997.

3. Giovanni Boccaccio, Genealogie deorum Gentilium, a cura di V. Zaccaria, Milano, Mondadori, I 998-99, 2 voll.

4. A questo proposito mi permetto di rinviare a C. Caruso, Adonis. The Myth of the Dying God in the Italian Renaissance, London, Bloomsbury, $2015^{2}$, pp. 6-20, da dove riprendo e sviluppo alcuni temi in questa sede.

5. Caruso, Adonis, cit., pp. 2 I-28.

6. Cfr., per esempio, Buccolicum carmen, I, 52; III, 9I; Xv, 9 (Giovanni Boccaccio, Buccolicum carmen, a cura di G. Bernardi Perini, in Giovanni Boccaccio, Tutte le opere, a cura di V. Branca, Milano, Mondadori, i994, v.2, pp. 656, 668, 878). Di grandissima utilità è la banca dati Poeti d'Italia in lingua latina (http://www.poetiditalia.it/, consultata il 4 ottobre 2016), che consente di rilevare con rapidità non solo le occorrenze del nome ma anche i relativi epiteti, che nel caso di Adone presentano un alto tasso di topicità (nella fattispecie delle tre occorrenze del Buccolicum carmen, rispettivamente: «iuvenis», «venans», «pulcher»).

7. Battista Spagnoli, detto il Mantovano, De calamitatibus temporum libri tres, a cura di G. Wessels, Roma, Tipografia Pontificia, I916, p. 46, e la sopra ricordata banca dati Poeti d'Italia in lingua latina (http://www.poetiditalia.it/).

8. Cfr. E. Carrara, La poesia pastorale, Milano, Vallardi, i936, p. 384.

9. Pierre de Ronsard, Adonis, in CEuvres complètes, a cura di J. Céard, D. Ménager e M. Simonin, Paris, Gallimard, I993-94, 2 voll., II, p. I397.

Io. M. Detienne, Les jardins d'Adonis. La mythologie des parfums et des aromates en Grèce [1972], Paris, Gallimard, $2007^{2}$, con postfazione dell'autore.

I I. G. Piccaluga, Adonis, i cacciatori falliti e l'avvento dell'agricoltura, in Il mito greco. Atti del Convegno internazionale (Urbino 7-12 maggio 1973), a cura di B. Gentili e G. Paioni, Roma, Edizioni dell'Ateneo e Bizzarri, I977, pp. 33-5 I; contra M. Detienne, Le chasseur malheureux, in "Quaderni urbinati di cultura classica», 24, I977, pp. 7-26. Per un più recente intervento sulla questione si veda $\mathrm{A}$. Testa, Miti antichi e moderne mitologie, Torino, Aragno, 2010, pp. 291-378.

i 2. Giovan Battista Marino, L'Adone, a cura di G. Pozzi [e collaboratori], Milano, Mondadori, 1976, 2 voll. (Milano, Adelphi, I988²).

13. A. Martini, Oltre l'idillio, in Lectura Marini, a cura di F. Guardiani, Ottawa, Dovehouse, I989, pp. 13-23, specialmente le pp. I4-I 5 . 
i 4. Lodovico Dolce, Stanze nella favola d'Adone, in appendice a Lodovico Dolce, Il Capitano. Comedia, Venezia, Gabriel Giolito de Ferrari, I 545, cc. 40v-57r; Giovanni Tarcagnota, L'Adone, in appendice a A. Borzelli, Il Cavalier Giambattista Marino, Napoli, Priore, I898, pp. 307-24; Girolamo Parabosco, Favola d'Adone, in Il terzo libro delle lettere amorose, Venezia, Griffio, I 558 , cc. 40v-5or. Si veda, in generale, G. Tallini, La 'Favola d'Adone' da G. Tarcagnota a G.B. Marino. Studi sulla letteratura regionale del basso Lazio tra Rinascimento e Barocco, Pescara, Libreria dell'Università, 2002, con il suo più recente Ludovico Dolce, Giovanni Tarcagnota e Girolamo Parabosco. Stanze nella favola d'Adone, Roma, Aracne, $20 \mathrm{I} 2$ (in base al quale ho qui corretto le date delle prime edizioni dei tre testi rispetto a Caruso, Adonis, p. 32). Sull'Adone del Dolce si veda A. Torre, Come lavorava Lodovico Dolce, in Dissonanze concordi. Temi, questioni e personaggi intorno ad Anton Francesco Doni, a cura di G. Rizzarelli, Bologna, Il Mulino, 2013 pp. 229-54; A. Torre, L'edonista riluttante, in «Italique», I7, 2014, pp. 75-101.

I 5. Come già osservava D.A. Stone Jr., 'L'Adonis' de Ronsard et Andrea Navagero, in «Bibliothèque d'humanisme et Renaissance», 43, I98 I, pp. I $55-58$. Il testo è quello della moderna edizione (con traduzione) a cura di A.E. Wilson: ANDrEA Navagero, Lusus, Nieuwkoop. B. de Graaf, i 973, p. 40.

i6. Giangiongio Trissino, La quinta e la sesta divisione della Poetica, in Trattati di poetica e retorica del Cinquecento, a cura di B. Weinberg, Bari, Laterza, I 970-74, 4 voll., II, p. 88: «la rima [...] al parlare rustico e pastorale non ben si conviene». Si veda P. Cosentino, Una "zampogna tosca" alla corte di Francia: le egloghe in versi sciolti di Luigi Alamanni, in «Filologia e critica», 28, 2003, pp. 70-95.

17. Alamanni, Opere toscane, Lione, Sebastianus Gryphius, I 532-33, 2 vol., I, pp. I 5 5-60, ristampato in Alamann, Versi e prose, a cura di P. Raffaelli, Firenze, Le Monnier, I 859, 2 voll., I, pp. 27-3 I; Girolamo Muzio, Egloghe del M. Iustinopolitano divise in cinque libri, Venezia, Gabriel Giolito de Ferrari, I 550, cc. Iorv-ıosr. Sulla composizione della Venere del Muzio si veda L. Borsetto, Lettere inedite di Girolamo Mu₹ৃio tratte dal codice Riccardiano 2IIs, in «Rassegna della letteratura italiana», 94, 1990, pp. 99-179 (a p. I60); L. Borsetto, L'egloga in sciolti nella prima metà del Cinquecento. Appunti sul 'liber' di Girolamo Mu₹rio, in Miscellanea di studi in onore di Giovanni da Požo, a cura di D. Rasi, Roma-Padova, Antenore, 2004, pp. I 23-6 I.

I 8. Adone è prole del rapporto incestuoso tra Mirra e il padre Cinira, a sua volta nipote di Pigmalione.

19. Mi permetto per questo di rinviare a Caruso, Adonis, cit., pp. 28-32, con relativa bibliografia.

20. Cfr. Caruso, Adonis, cit., pp. 31-32.

2i. Pierre de Ronsard, Elegie sur le livre de la chasse du feu Roy Charles IX, in Euvres complètes, I, p. I I 54; Claude Binet, Adonis, ou le Trespas du Roy Charles IX. Eglogue de Chasse, in Merveilleuse Rencontre sur les noms tournez du Roy et de la Royne, Paris, Fédéric Morel, i 575, pp. 8-9; Guillaume Le Breton, Adonis. Tragedie françoyse (I 579), in Gabriel Le Breton, Adonis, a cura di M. Bensi, in Théâtre français de la Renaissance. La tragédie à l'époque d'Henri III. Deuxieme Série, I 574-79, Firenze-Paris, OlschkiPUF, I 999, 5 voll., I (permane qualche incertezza riguardo il nome di Le Breton). 


\section{Carlo Caruso}

22. Giulio Cesare Scaligero, Poetices libri septem, a cura di L. Deitz e G. VogtSpira, con la collaborazione di M. Fuhrmann, Stuttgart-Bad Cannstatt, Frommann-Holzboog, I 994-2003, 5 voll., I, p. 94.

23. Gabriel Le Breton, Adonis. Tragedie françoyse, Rouen, Raphaël du Petit Val, i6 II.

24. Su Enrico IV come Pan cfr. Recueil de diverses poesies sur le trespas de Henry le Grand thres-chrestien Roy de France \&o de Navarre, et sur le sacré et couronnement de Louis XIII, son successeur. Dedié a la Royne, Mere du Roy, Regente en France, a cura di Guillaume Dupeyrat, Paris, Robert Estienne e Pierre Chevalier, I6 I I, cc. 24v e 90r-97v. Ravaillac è paragonato al cinghiale alle cc. Io6r-8v: cfr. in particolare l'espressione «triste bidental» (c. I07r), con riuso e variatio di Orazio, Ars poetica, $47 \mathrm{I}$.

25. Caruso, Adonis, cit., pp. 86-90.

26. Sulla tradizionale associazione di Adone alla regalità attraverso il topos della bellezza del sovrano si veda P. Cherchi, Il re Adone, Palermo, Sellerio, I999.

27. Camillo Pellegrino, Primicerio di Capua, lettera ad Alessandro Pera della fine del i 5 96: «Ho inteso far le meraviglie del poema d'Adone, che il signor Marino ha per le mani» (citato in Borzelli, Il Cavalier Giambattista Marino, cit., p. 2 Io).

28. Cfr. Giovan Battista Marino, La sampogna, a cura di V. De Maldé, MilanoParma, Fondazione Bembo-Guanda, I993, p. xcIv.

29. Si veda la recentissima edizione critica: Giovan Vincenzo Imperiali, Lo stato rustico, a cura di O. Besomi, A. Lopez-Bernasocchi e G. Sopranzi, Roma, Edizioni di Storia e Letteratura, 20I 5 , 2 voll.

30. Giovan Battista Marino, Lettere, a cura di M. Guglielminetti, Torino, Einaudi, I966, pp. 204, 24I. Due degli editori che collaborarono all'edizione dell'Astrée, Toussain de Bray e Olivier de Varennes, pubblicarono nei medesimi anni rispettivamente la Sampogna (I620) e l'Adone (I623). Per la complessa storia delle edizioni dell'Astrée cfr. Honoré D’Urfé, L'Astrée, a cura di H. Vaganay, Lyon, Pierre Masson, I 925-28, 5 voll., v, pp. 55 I-6I. Sulla varietà degli esemplari dell'Adone cfr. Giambonini, Bibliografia delle opere a stampa di Giambattista Marino, Firenze, Olschki, 2000, 2 voll, I, cat. n. I e 2.

3i. Giovan Battista Marino, Adone, a cura di E. Russo, Milano, BUR, 20 3, i, pp. I 8-22.

32. Incertezze chiaramente attestate nel suo epistolario. Cfr. per esempio la lettera da Parigi a Fortuniano Sanvitale datata genericamente i620 nell'ed. Guglielminetti, che è quasi certamente della seconda metà del luglio di quell'anno: «[...] e già tutta la Francia è in guerra. [...] se le cose andassero contrarie per alcuni personaggi, che al presente sono in favore ed in grandezza, sarei costretto a mutar nel libro molte circostanze particolari» (MARINO, Lettere, p. 283 ). Cfr. E. Russo, Marino, Roma, Salerno, 2008, p. 259. 